\title{
Equations of relative pressure losses in processes of suction and injection of piston pump
}

\author{
V.E. Shcherba \\ Doctor of Technical Sciences, Professor \\ Omsk State Technical University, Russia \\ Scherba_V_E@list.ru
}

\section{G.S. Averyanov}

Doctor of Technical Sciences, Professor

Omsk State Technical University, Russia, Scherba_V_E@list.ru

\author{
V.V. Shalai \\ Doctor of Technical Sciences, Professor \\ Omsk State Technical University, Russia \\ Scherba_V_E@list.ru
}

\author{
S.V. Korneev \\ Doctor of Technical Sciences, Professor \\ Omsk State Technical University, Russia \\ Scherba_V_E@list.ru
}

\begin{abstract}
The article presents analytical equations for determining the relative pressure losses in suction and discharge processes, both including and excluding the liquid compressibility; they were obtained based on an analysis of the approach to determine the relative pressure losses in the suction and discharge processes in a piston compressor. The numerical experiment has confirmed their applicability.
\end{abstract}

Keywords- piston pump, relative suction pressure, relative discharge pressure, energy conservation equation, equation of motion, piston, cylinder.

\section{INTRODUCTION}

A significant amount of energy is currently being spent for the pump drives in all countries. The amount of energy consumed in different countries is different and, according to experts' estimates, reaches up to $20 \%$ of all generated electricity [1]. For example, about $12 \%$ of all electricity generated is spent on pump drives in the industrial sector in Germany.

Piston pumps, including axial-piston and radial-piston pumps, have a significant part both in quantity and in energy consumption among the existing pumps; they are absolute leaders in the volume pumps and are widely used in the aviation industry. The suction and discharge processes are the most important in the cycle of a volume pump, as they provide the bulk of the technical work, and their quality largely determines the pump energy efficiency, and its uneven supply.
Due to the above said, the relevance of this work is beyond doubt.

\section{THEORETICAL BASES OF THE ANALYSIS METHODS}

Differential equations of relative pressure losses in the suction and discharge processes for piston compressors were obtained by academician N.A. Dollezhal [2]. To calculate the absorption process, he used the first law of thermodynamics for working medium with variable mass and without external heat exchange and flash gas through the piston seal leakage, the equation of an incompressible flow of gas through the hole and the equation of ideal gas state. The equation of adiabatic compression $p v^{k}=$ const was applied for the injection process and it can be obtained applying the first law of thermodynamics to the working medium with variable mass and without external heat exchange and flash gas [3], the equation of gas flow through a discharge valve assuming its incompressibility, and the equation of ideal gas state.

Thus, Academician N.A. Dollezhal used two basic equations of energy conservation:

- internal energy - in the form of the first law of thermodynamics for working medium with a variable mass;

- external energy - in the form of the Bernoulli equation for the flow of an incompressible fluid (the equation for the flow of incompressible fluid through an aperture is derived from the Bernoulli equation) assuming that working medium is ideal gas.

The system of three equations with three unknown $p, T, \rho$ (where $p$ is pressure, $T$ is temperature, $\rho$ is 
density) was solved with respect to dimensionless pressure losses $\chi$ : for the suction process $-\chi_{s c}=\frac{p_{s c}-p}{p_{s c}}$, for the injection process $-\chi_{i j}=\frac{p_{i j}-p}{p_{i j}}$, where $p_{s c}, p_{i j}$ are nominal pressures in the process of suction and injection.

The authors, considering calculations of suction and discharge processes in volumetric pump, apply the first law of thermodynamics to the working medium with variable weight for a stream, which hereinafter also is converted into a system of differential equations of internal and external energy conservation [4].

Thus, there is regularity of the fact that to obtain the pressure loss equations (in absolute or relative form), it is necessary to apply:

- The first law of thermodynamics to the working medium with a variable weight as an internal energy conservation law.

- The Bernoulli equation as a law of mechanical energy conservation.

- The closing equation in the form of the equation of state for gas and Hooke's equation for a dropping liquid.

Little effect of external heat transfer during the volumetric pumps should be noted; at high heat capacity, droplet does not lead to noticeable change in its temperature. Thus, when determining equations, pressure losses in positive displacement pump can be assumed $T=$ const, and when analyzing suction and injection processes, the equation of the first law of thermodynamics can be excluded from consideration.

\section{SUCTION PROCESS}

In general, the flow of fluid in the suction pipeline and in the pump cylinder is one-dimensional and non-stationary, because the speed of the piston is variable. A system of differential equations describing a nonstationary onedimensional flow and including the equation of motion and the equation of continuity can be represented in the form [5]:

$$
\left\{\begin{array}{l}
\rho \frac{\partial Q}{d t}+F \frac{\partial p}{\partial x}+\frac{\lambda g}{2 d F} Q|Q|=0 \\
\frac{g c^{2}}{F} \cdot \frac{\partial p}{\partial x}+\frac{\partial p}{\partial t}=0
\end{array},\right.
$$

where $p, Q$ are pressure and volumetric flow of liquid in the pipeline; $F$ is the cross-sectional area of the pipeline; $d$ is its diameter; $x, t$ are time and coordinate; $\lambda$ is coefficient of friction along the length; $\rho, c$ are fluid density and the speed of sound in it.

The system of differential equations $(1,2)$ in separate derivatives has no analytical solution and may be solved with special methods such as the method of characteristics [6].

When analyzing the suction process of the low pressure and low pressure drops due to change in droplet, density can be neglected and it is assumed that $\rho=$ const. Then the unknown variable is one $p(\rho=$ const, $T=$ const $)$ at a known value of the velocity $v$ along the pipeline axis. In this case, a quasi-stationary model of fluid flow in a suction pipeline is to be accepted, and the Bernoulli equation for the viscous fluid flow to determine the relative pressure losses during the suction process is to be used [7]; its differential form is [8]:

$g d z+\frac{d p}{\rho}+v d v+g d h w=0$.

Considering that $\rho=$ const, the cross-section I-I is located at the free liquid surface in the suction tank, and the cross section II-II is located on the bottom of the piston (Fig.1), equation (3) integrating gives the following mathematical equation:

$z_{1}+\frac{p_{1}}{\rho g}+\frac{\alpha_{1} v_{1}^{2}}{2 g}=z_{2}+\frac{p_{2}}{\rho g}+\frac{\alpha_{2} v_{2}^{2}}{2 g}+\left(\sum \lambda_{i} \frac{\ell_{i} f_{i}^{2}}{d_{i} f_{n}^{2}}+\sum \zeta_{i}\right) \frac{v_{2}^{2}}{2 g}$.

In most cases, there are $\mathcal{V}_{1}=0 ; \alpha_{2}=1 ; p_{1}=p_{s c} ; p_{2}=p$; $v_{2}=v_{h}$. Considering the above said transformation results, there is an equation:

$p_{s c}-p=\left(z_{2}-z_{1}\right) \rho g+\left(1+\sum \lambda_{i} \frac{\ell_{i}}{d_{i}} \frac{f_{i}^{2}}{f_{n}^{2}}+\sum \zeta_{i}\right) \frac{v_{n}^{2}}{2 g} \rho g$,

or

$\chi_{s c}=\frac{\rho g}{p_{s c}}\left[\left(z_{2 o}-S n-z_{1}\right)+\left(1+\sum \lambda_{i} \frac{\ell_{i}}{d_{i}} \frac{f_{i}^{2}}{f_{n}^{2}}+\sum \zeta_{i}\right) \frac{v_{n}^{2}(\varphi)}{2 g}\right]$,

where $\operatorname{Sn}(\varphi)=S_{M}+\frac{S h}{2}\left[(1-\cos \varphi)+\frac{\lambda_{0}}{4}(1-\cos 2 \varphi)\right]$ are the current values of the stroke of the piston; $S_{M}$ is the value of the linear dead volume; $z_{2 o}, z_{1}$ are coordinates of the piston in TDC, coordinates of the free surface of the liquid in the suction tank; $\lambda_{i}$ is the value of the friction coefficient along the length at the $\mathrm{i}$-th section of the suction line; $\ell_{i}, d_{i}$, $f_{i}$ are, respectively, the length, diameter and cross-sectional area of the $\mathrm{i}$-th section of the pipeline; $\sum \zeta_{i}$ is the sum of local coefficients of resistance, reduced to the speed of the piston $\left(v_{n}\right) ; v_{n}=\frac{S h}{2} \omega\left(\sin \varphi+\frac{\lambda_{0}}{2} \sin 2 \varphi\right)$ is speed of the piston; $\omega$ is angular speed of the crankshaft rotation.

Analyzing the structure of equation (6), the following conclusions are offered:

1. The first term determines part of the pressure losses, connected with the fluid lifting from the free surface to the bottom of the piston.

2. The second term shows the pressure losses for overcoming the hydraulic resistances and for obtaining fluid velocity equal to the velocity of the piston.

\section{INJECTION PROCESS}

In general, the pressure $p$ is a function of the specific volume $v$ and temperature, but, considering that the temperature is constant, it is possible to write it as follows: 
$\beta p=\frac{1}{E}=-\frac{1}{v} \frac{d v}{d p}$

thus $d p=E \frac{d \rho}{\rho}$.

Considering equation (8), the Bernoulli equation takes the form:

$$
z+\int \frac{d p}{\rho}+\frac{\alpha v^{2}}{2 g}=z+\int \frac{E}{\rho^{2}} d \rho+\frac{\alpha v^{2}}{2 g}=\text { const } .
$$

After integration, equation (9) is transformed to the following form:

$z_{1}-\frac{E}{\rho_{1} g}+\frac{\alpha_{1} v_{1}^{2}}{2 g}=z_{2}-\frac{E}{\rho_{2} g}+\frac{\alpha_{2} v_{2}^{2}}{2 g}+\Delta h w_{H}$.

Parameters with index "1" refer to the cross-section I-I, and it is connected with the bottom of the piston to determine the relative pressure losses during injection, and parameters with index "2" - to cross-section II-II, located immediately behind the discharge valve in the discharge line (Fig.1).

Equation (8) integrating gives the formula:

$\rho_{2}=\rho_{1} \exp \left(\frac{p_{2}-p_{1}}{E}\right)$.

Substituting equation (11) into equation (10) results in:

$p_{1}-p_{2}=E \ell n\left\{1+\frac{\rho_{1} g}{E}\left[\left(z_{2}-z_{1}\right)+\left(\frac{\alpha_{2} v_{2}^{2}}{2 g}-\frac{\alpha_{1} v_{1}^{2}}{2 g}\right)+\Delta h w_{u}\right]\right\}$.

And it represents the following equation in the dimensionless form:

$\chi_{u}=\frac{E}{p_{u}} \ln \left\{1+\frac{\rho_{1} g}{E}\left[\left(z_{2}-z_{1}\right)+\left(\frac{\alpha_{2} v_{2}^{2}}{2 g}-\frac{\alpha_{1} v_{1}^{2}}{2 g}\right)+\Delta h w_{H}\right]\right\}$,

where $z_{1}, z_{2}$ are the coordinates of the centers of gravity of the corresponding sections.

$z_{1}=z_{n}, z_{2}=z_{\mu}$ where $z_{n}$ is coordinate of the bottom of the piston; $z_{\mu}$ is the coordinate of the section gravity; $\mathrm{p}_{\mathrm{ij}}$ is nominal discharge pressure; $v_{1}=v_{n}$ is speed of the piston; $v_{2}=\frac{v_{1} f_{n}}{f_{H}}$ is velocity of the fluid in section II-II; $f_{t}$ is the cross-sectional area of the injection pipeline; $\alpha_{2}, \alpha_{1}$ are values of Coriolis coefficients in the corresponding sections (values $\alpha_{1}$ and $\alpha_{2}$ can be taken as 1 ).

The amount of hydraulic resistance in the discharge line $\Delta h w_{H}$ is composed of hydraulic resistances along the length $\Delta h \boldsymbol{H} \ell$ and local hydraulic resistances. Their meaning is determined with the formulas:

$$
\begin{aligned}
\Delta h_{\zeta \mu} & =\zeta_{\text {val }} \frac{v_{2}^{2}}{2 g} ; \\
\Delta h_{\ell H} & =\lambda \frac{\operatorname{Sn}(\varphi)}{d} \frac{v_{1}^{2}}{2 g},
\end{aligned}
$$

where $\zeta_{v a l}=\left(\frac{f_{u}}{f_{g}}-1\right)^{2}$ is a coefficient of local resistance of the

valve; $f_{g}$ is gap area of the discharge valve; $\lambda$ is coefficient of friction along the length (generally is a function of the Reynolds number and relative roughness and is determined according to the existing recommendations [9,10 and so on]).

The structure of the relative pressure losses during the injection process, that were obtained considering the compressibility of the liquid, is similar to the structure of the relative pressure losses during the suction process.

\section{THE RESULTS ANALYSIS}

The calculation of relative pressure losses in the suction and discharge processes of a single-cylinder piston pump, acting and having the following parameters, was carried out:

nominal suction pressure $\left(p_{s c}\right)$ is $0.1 \mathrm{MPa}$;

nominal discharge pressure $\left(p_{d}\right)$ is $20 \mathrm{MPa}$;

diameter of the cylinder is $50 \mathrm{~mm}$;

stroke of the piston is $50 \mathrm{~mm}$;

crankshaft rotation speed is $250 \mathrm{~min}^{-1}$;

center of gravity of sections: $z_{s c}=1 \mathrm{~m} ; z_{20}=2 \mathrm{~m} ; z_{d}=2.2 \mathrm{~m}$;

working medium - water with the following properties: $E=2 \cdot 10^{9} \mathrm{~Pa} ; \rho_{0}=1000 \frac{\mathrm{kg}}{\mathrm{m}^{3}}$ at $T_{0}=293 \mathrm{~K}, p_{0}=0.1 \mathrm{MPa}$;

$\sum \ell_{i}=\ell_{i j}=1 m$ is length of the suction pipe;

$\sum \zeta_{i}=\zeta_{\mathrm{csc}}=5$ is the sum of local coefficients of resistance at suction;

$d_{s c}=20 \mathrm{~mm}$ is a diameter of the suction pipe;

$d_{d}=20 \mathrm{~mm}$ is a diameter of the discharge pipeline;

$S_{H}=1 \mathrm{~mm}$ is linear dead space;

$\zeta_{\text {val }}=5$ is a value of the local coefficient of resistance of the discharge valve.

The relative pressure losses in the suction and discharge processes at different angular velocity and discharge pressure are shown in Fig. 2, 3 and 4. The presented results draw the following conclusions:

1. As the angular velocity of rotation increases, the relative pressure losses increase both during the suction process and during the injection process. The maximum values of the relative pressure losses are achieved in the middle of the processes, when the piston has its maximum speed.

2. With increasing injection pressure, the density of the working fluid increases, and it leads to an increase in the absolute values of the pressure losses. However, the relative value of the pressure losses decreases; the increase in pressure itself exceeds the increase in pressure loss.

\section{CONCLUSION}

Mathematical dependencies are obtained to calculate the relative pressure losses in the suction and discharge processes for a piston pump. For the injection process, the changes in the density of the working medium on the pressure are considered. The numerical experiment showed the applicability of the equations obtained. 


\section{REFERENCES}

[1] T. Hinenger, R. Schmidt-Fallus, M. Norden, Optimization of processes due to self-tuning in pumping systems is one of the possibilities for improving efficiency, Pumps, compressors, fittings, 8, pp.4-10, 2017.

[2] P.I. Plastinin, Reciprocating compressors, Vol. 1, M.: Kolos, 2006, pp.397.

[3] V.E. Shcherba, Working processes of compressors of volumetric action, M.: Nauka, 2008, pp.319.

[4] V.E. Shcherba, V.V. Shalai, A.V. Grigoriev, Generalized thermodynamic approach to the calculation of suction and discharge processes in pumps and compressors of volumetric action, Chemical and oil and gas engineering, №6, pp.26-29, 2015.
[5] A.G. Gimadiev, N.D. Bystrov, Dynamics and regulation of hydraulic and pneumatic systems, Samara, 2010, pp.178

[6] V.S. Gavrilov, N.A. Denisova, Method of characteristics for onedimensional wave control, Nizhny Novgorod, 2014, pp.72.

[7] A.G. Skhirtladze, V.I. Ivanov, V.N. Karev [et.al.], Hydraulics in mechanical engineering, Stary Oskol: TNT, 2008, Part 1, pp.392

[8] A.D. Altshul, P.G. Kiselev, Hydraulics and aerodynamics, M.: Stroyizdat, 1975, pp. 327.

[9] I.E. Idelchik, Reference book on hydraulic resistance, M.: 2012, pp.466.

[10] A.V. Lepeshkin, A.A. Mikhaylin, Hydraulic and pneumatic systems, Moscow: Publishing Center "Academy", 224, pp.336. 